\title{
CONTROLLER REDUCTION METHODS MAINTAINING PERFORMANCE AND ROBUSTNESS
}

John B. Moore and Andrew J. Telford

Department of Systems Engineering, Research School of Physical Sciences,

Australian National University, P.O. Box 4, Canberra, ACT 2601, Australia.

Uy-Loi Ly

The Boeing Company, Seatte, Washington U.S.A.

ABstract

In reducing high order controller designs, such as arise from $H^{\infty}$ or LQG techniques, to more practical low order ones, a reasonable objective is to preserve the controller robustness/performance properties. In this paper, standard balanced truncation or Hankel norm model approximation methods are applied to augmentations of the controller which emerge when characterizing the class of all stabilizing controllers in terms of an arbitrary proper stable transfer function.

In the method, scaling parameters are at the disposal of the engineer to achieve an appropriate compromise between preserving performance for the nominal plant and a certain type of robustness to plant variations. There are a number of unique features of the approach of the paper. One feature is that a straightforward re-optimization of a reduced-order controller is possible within the framework of the method. A second feature of the paper is that for controllers designed for simultaneous stabilization of a number of plants, the method seeks to preserve the performance/robustness of the reduced order controller for each plant.

\section{INTRODUCTION}

The model reduction methods of [1] provide a priori bounds on reduction errors in terms of $L^{\infty}$ measures. A simpler technique, termed balanced realization, has guaranteed bounds which are not quite so good, see also [1], [2]. Such techniques are then attractive to achieve controller reduction, but without modification do not take into account the fact that the controller is in a control loop and needs to achieve performance and robustness properties. In the reduction, these techniques without modification weight all frequencies equally.

The notion of a frequency-wcighted model reduction based on the techniques of $[1],[2]$ has been explored in $[3],[4],[5]$. It is not clear from these results how best to use knowledge of the frequency characteristics of a plant, or closed-loop, to frequency-weight the controller reduction. Special frequencyweightings based on controller characteristics are studied in (5).

A technique for controller reduction for linear quadratic gaussian designs is given in [6]. This exploits the fact that the innovations process is white - (as in the techniques of [7]) and reduces the subsystems of the controller driven from this white noise. In effect there is a particular coprime stable factorization of the controller, and it is proposed that reductions on these be implemented using standard methods (balanced realizations without frequency shaping). A possible disadvantage for this approach is that stability of the original controller design is not guaranteed in the reduction.

In this paper, a novel controller reduction approach is proposed. It is based on the application of standard model reduction techniques to a system calculated from both plant and controller. The method utilizes theory for the class of all stabilizing controllers [8] based on the work of [9]. Thus referring to Figure 1.1 with plant $G(s) \in R_{p}$, controller $K(s) \in R_{p}$, then the class of all stabilizing controllers is given in terms of $J(K, G) \in R_{\mathrm{p}}$ and arbitrary $Q(s) \in$ $R H^{\infty}$, where $R_{p}$ denotes the class of rational proper transfer functions and $R H^{\infty}$ the class of stable rational proper transfer functions

The selection of $J(s)$ we consider is where the block $J_{11}(s)$ is in fact the controller $K(s)$, and the other elements $J_{i j}(s)$ are appropriately scaled. Using the circumflex to denote a low order approximation, we propose that $J(s)$ first be approximated by $\hat{J}(s)$ using standard model reduction (possibly frequency-weighted). Then the reduced order controller $\hat{K}(s)$ is taken as the 11-block of this. That is,

$$
\hat{K}(s)=[\hat{J}(s)]_{11} \text { where } K(s)=J_{11}(s)
$$

This contrasts the more direct application of model reduction where only $J_{11}(s)=K(s)$ is approximated, so that $\hat{K}(s)=j(s)_{11}$.

An extension of the approach proposed is to work with the class of controllers of Figure $1.1 \mathrm{~b}$ with $Q(s)$ constrained as constant. Thus consider the

Work partially supported by Defense Science and Technology Organization, Ausiralia.
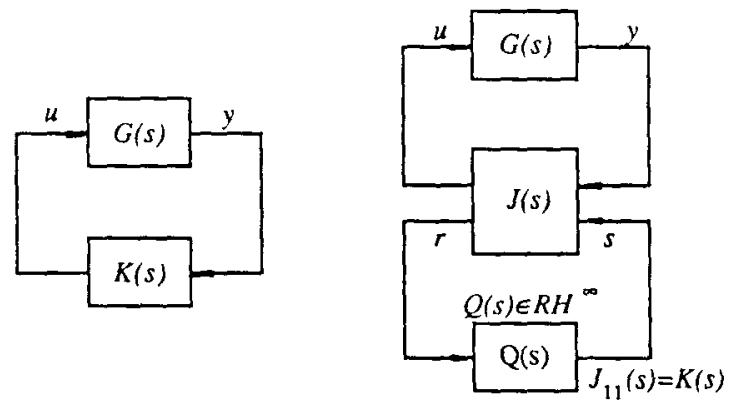

(a) Closed-loop system

(b) Class of all stabilizing controllers $K(Q, s)$

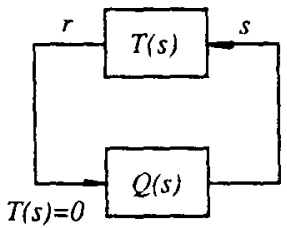

(c) a reorganization

Figure 1.1

class

$$
K(Q, s)=J_{11}(s)+J_{12}(s) Q\left[I-J_{22}(s) Q\right]^{-1} J_{21}(s), Q \text { constant }
$$

and its reduced order versions

$$
\left.\dot{K}(Q, s)=[j(s)]_{11}+[\hat{J}(s)]_{12} Q\left[I-[\hat{J}(s)]_{22} Q\right]^{-1}[\hat{J}(s)]\right]_{21}
$$

Ilere $\hat{K}(s)$ or $(1.1)$ is equal to $\hat{K}(Q=0, s)$. Also note that

$$
\text { degree } \hat{K}(Q \mid Q=\text { constant }, s)=\operatorname{degree} \hat{K}(Q=0, s)
$$

In this paper one proposal is that $\hat{K}(Q \mid Q=$ constant, $s)$ be re-optimized over constant $Q$ in terms of the original (or related) controller robustness/performance objectives.

To maintain performance which penalizes some internal variables, or their estimates $e$, a refinement of the above method is to modify the $J(s)$ or $K(Q, s)$ blocks in Figure 1.1 to have an additional output $c$. Denoting these blocks as $J_{e}(s), K_{e}(Q, s)$ we propose the reduction of $K(s)$ via $J_{e}(s)$ or $K,(Q, s)$ to maintain performance as well as robustness. Again scaling gives desired trade-off between performance and robustness. When $K(s)$ is designed to achieve simultaneous stabilization of a number of plants, it is proposed to maintain its performance/robustness properties for each of these plants by working with appropriate augmentations of $J(s)$. Details are given in the paper. A dual version of the method is where the role of $G(s)$ and $K(s)$ are interchanged.

In the next Section 2, the controller reduction techniques for preserving robustness are given in details. A rationale for the proposed controller reduction is given in Section 3, and examples are studied in Section4. Conclusions are drawn in Section 5.

\section{DETAILS OF CONTROLLER IEDUCTION}

\subsection{Definitions}

Referring to Figure 1.1, let us first recall the formulation of $J_{11}(s)=K(s)$ based on the theory for the class of all stabilizing controllers [12]. Lct us 


$$
G(s)=C(s I-A)^{-1} B+D=\left[\begin{array}{l|l}
A & B \\
\hline C & D
\end{array}\right]_{T}
$$

Also, in the first instance let us consider that $K(s)$ belongs to the stabilizing controller class having the form of Figure 2.1 for the case $Q(s) \equiv 0$. Thus $K(s)$ is characterized in terms of $F, H$ (see also Section 2.7) as

$$
\begin{aligned}
K(s)= & {\left[\begin{array}{c|c}
A^{*} & -H \\
\hline F & 0
\end{array}\right]_{T} \in R_{s p}, \quad A^{*}=A+B F+H C+H D F } \\
& {[s I-(A+B F)]^{-1},[s I-(A+H C)]^{-1} \in R H^{\infty} }
\end{aligned}
$$

where $R_{s p}$ denotes rational strictly proper.

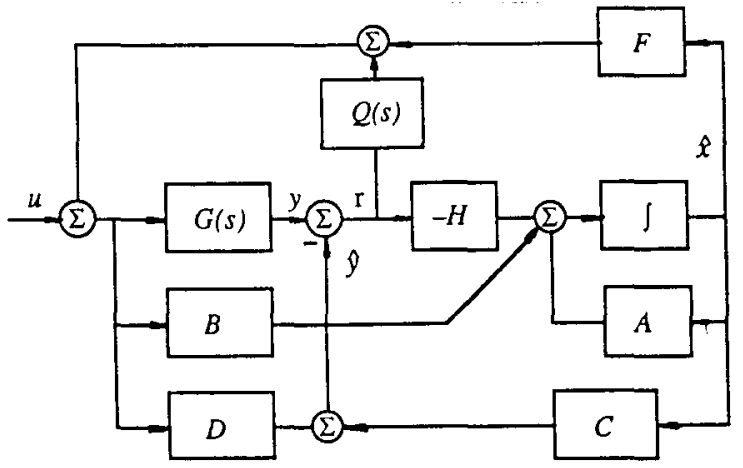

Figure 2.1 Controller class

Clearly, the class of LQG controllers is a subset of this controller class. From [12], the class of all stabilizing controllers for $G(s)$ has the form of Figure 2.1, being parameterized in terms of $Q(s) \in R H^{\infty}$. Moreover $J(s)$ has the form

$$
J(s)=\left[\begin{array}{c|cc}
A^{*} & -H & B+H D \\
\hline F & 0 & I \\
-C-D F & I & -D
\end{array}\right]_{T}, J_{11}(s)=K(s)
$$

It should be clear that Figure 1.1b for this case takes the form of Figure 2.1 with the $Q(s)$ nonzero.

Other relationships of interest are reviewed. Defining

$$
\begin{aligned}
& x \triangleq\left[\begin{array}{ll}
M(s) & U(s) \\
N(s) & V(s)
\end{array}\right]=\left[\begin{array}{ccc}
A+B F & B & -H \\
\hline F & I & 0 \\
C+D F & D & I
\end{array}\right]_{T} \in R H^{\infty} \\
& \tilde{X} \triangleq\left[\begin{array}{cc}
\tilde{V}(s) & -\dot{U}(s) \\
-\tilde{N}(s) & \tilde{M}(s)
\end{array}\right]=\left[\begin{array}{c|cc}
A+H C & -(B+H D) & H \\
\hline F & I & 0
\end{array}\right]_{T} \in R H^{\infty}
\end{aligned}
$$

then

$$
\tilde{X}(s) \mathcal{X}(s)=\lambda(s) \cdot \overline{\mathcal{X}}(s)=I \quad \text { (double Bezout) }
$$

$G(s)=N(s) M(s)^{-1}=\tilde{M}(s)^{-1} \tilde{N}(s), \quad K(s)=U(s) V(s)^{-1}=\tilde{V}(s)^{-1} \tilde{U}(s)$

Also

$$
\begin{aligned}
J(s)= & {\left[\begin{array}{ll}
J_{11}(s) & J_{12}(s) \\
J_{21}(s) & J_{22}(s)
\end{array}\right]=\left[\begin{array}{cc}
K(s) & \tilde{V}(s)^{-1} \\
V(s)^{-1} & -V(s)^{-1} N(s)
\end{array}\right] } \\
& =\left[\begin{array}{cc}
K(s) & M(s)-K(s) N(s) \\
\tilde{M}(s)-N(s) K(s) & -[\bar{M}(s)-\tilde{N}(s) K(s)] N(s)
\end{array}\right]
\end{aligned}
$$

and referring to Figure $1.1 \mathrm{c}$,

$$
T(s)=J_{22}(s)+J_{21}(s) G(s)\left[I-J_{11}(s) G(s)\right]^{-1} J_{12}(s)=0
$$

\subsection{Scaling}

Before applying any multivariable model reduction technique o $J(s)$ to yield a $\hat{J}(s)$, it makes sense to scale the inputs $y(t), s(t)$ and outputs $u(t), r(t)$ in such a way that they are given appropriate significance. We

$t$ The top left, top right, bottom left and bottom right entries of the partitioned matrix in (2.1) represent the system matrix, input matrix, oulput matrix and direcl-fecdthrough matrix of a state-space realization of $J(s)$. The matrix subscript $T$ indicates that this notation is being used. do not propose an optimal scaling selection. Based on experience we know that scaling can be crucial to a good reduction. In the examples studied in this paper, we determine the scalings of the variables $y(t), s(t), u(t)$ and $r(t)$ using their closed-loop auto-covariance responses to realistic stochastic disturbances. This is achieved by solving a steady state Lyapunov equation associated with the closed-loop system. Thus consider the stochastic closedloop system driven by the process noises $w(t)$ and measurement noise $v(t)$,

$$
\begin{aligned}
& \frac{d x(t)}{d t}=A x(t)+B u(t)+w(t) \\
& \frac{d \hat{x}(t)}{d t}=A \hat{x}(t)+B u(t)-H r(t)
\end{aligned}
$$

where $r(t)=y(t)-[C \dot{x}(t)+D u(t)], y(t)=C \cdot x(t)+D u(t)+v(t), u(t)=F \dot{x}(t)$

$$
E\left[\boldsymbol{w}(t) w^{T}(\tau)\right]=Q_{w} \delta(t-\tau), E\left[v(t) v^{T}(\tau)\right]=Q_{v} \delta(t-\tau)
$$

The state/state-estimate auto-covariance matrix $P$ satisfies the following Lyapunov equation.

$$
P A_{c}^{T}+A_{c} P+\left[\begin{array}{cc}
I & 0 \\
0 & -H
\end{array}\right] Q_{n}\left[\begin{array}{cc}
I & 0 \\
0 & -H
\end{array}\right]^{T}=0
$$

where

$$
P=E\left[\left(\begin{array}{l}
x \\
\dot{x}
\end{array}\right)\left(\begin{array}{l}
x \\
\dot{x}
\end{array}\right)^{T}\right], A_{c}=\left[\begin{array}{cc}
A & B F \\
-H C & A+B F+H C
\end{array}\right], Q_{n}=\left[\begin{array}{cc}
Q_{w} & 0 \\
0 & Q_{v}
\end{array}\right]
$$

and

$$
\begin{gathered}
E\left[y(t) y^{T}(t)\right]=\left[\begin{array}{lll}
C & D F
\end{array}\right] P\left[\begin{array}{ll}
C & D F
\end{array}\right]^{T}+\left[\begin{array}{lll}
0 & I
\end{array}\right] Q_{n}\left[\begin{array}{ll}
0 & I
\end{array}\right]^{T} \\
E\left[u(t) u^{T}(t)\right]=\left[\begin{array}{lll}
0 & F
\end{array}\right] P\left[\begin{array}{ll}
0 & F
\end{array}\right]^{T} \\
E\left[r(t) r^{T}(t)\right]=\left[\begin{array}{lll}
C-C & -C
\end{array}\right] P\left[\begin{array}{ll}
C & -C
\end{array}\right]^{T}+\left[\begin{array}{ll}
0 & I
\end{array}\right] Q_{n}\left[\begin{array}{ll}
0 & I
\end{array}\right]^{T}
\end{gathered}
$$

It is not possible to calculate a value for $E\left[s(t) s^{T}(t)\right]$, because it is dependent on the value of $Q(s)$. Choosing a value that it too large will place too much emphasis on the $s(t)$ input in the reduction. One suggested selection is to choose $E\left[s(t) s^{T}(t)\right]=E\left[u(t) u^{T}(t)\right]$. We propose that the square roots of the diagonal elements of these matrices be used to generate scaling matrices $D_{y}, D_{u}, D_{r}, D$, to scale $J(s)$ as follows

$$
J_{\text {caled }}(s)=\left[\begin{array}{c|cc}
A^{*} & -H D_{y} & (B+H D) D_{1} \\
\hline D_{u}^{-1} F & 0 & D_{u}^{-1} D \\
D_{r}^{-1}(C+D F) & D_{r}^{-1} D_{y} & -D_{r}^{-1} D D_{z}
\end{array}\right]_{T}
$$

In the system $J_{\text {ecaled }}(s)$ the variances of the scaled input/output variables in the closed-loop system will be unity.

Now model reduction techniques as in [1] can be applied to $J_{\text {scaled }}(s)$ to yield low-order models,

$$
\hat{J}_{\text {ccaled }}(s)=\left[\begin{array}{c|cc}
\widehat{\left(A^{*}\right)} & -\hat{W}_{\text {scaled }} & * \\
\hline \begin{array}{c}
\dot{F}_{\text {scaled }} \\
*
\end{array} & \begin{array}{c}
\dot{D}_{\text {scaled }} \\
*
\end{array}
\end{array}\right]_{T}
$$

from which a reduced order controller is taken as

$$
\hat{K}(s)=\left[\begin{array}{c|c}
\widehat{\left(A^{*}\right)} & -\hat{H}_{\text {ccaled }} D_{y}^{-1} \\
\hline D_{v} \hat{F}_{\text {callod }} & D_{u} \hat{D}_{\text {ccaled }} D_{y}^{-1}
\end{array}\right]_{T}=D_{u}\left[\hat{J}_{\text {cesied }}(s)\right)_{11} D_{y}^{-1}
$$

More generally, $\hat{K}(Q, s)$ can be in terms reductions on $J$ and constant $Q$ as in (1.3).

Other scaling possibilities can be envisaged. Observe that at the one extreme with $D_{u}$ approaching zero, then $[j(s)]_{11} \rightarrow j_{11}(s)$ and standard controller reduction is achieved. At the other extreme with $D_{r} \rightarrow 0$, maintaining prediction quality is emphasized-this is linked to maintaining quality of the state estimate feedback. When the prediction errors are white and state estimation is optimal, then with $D_{r} \rightarrow 0$ these qualities are preserved as much as possible.

Or course, a search procedure over $D_{u}, D_{r}$ and $D_{y}$ may achieve an improved compromise between performance and robustness. Such brute force optimizations are not explored further in this paper. There is no proof or rationale in this paper to suggest that a selection $D_{u} \neq 0$ is always better than a selection $D_{u}=0$. However, out experience has certainly shown that it is sometimes better. One scaling technique has been presented above based on certain intuitions which appear to work well. It could be used as the starting point for a search for an improved reduction. 


\subsection{Re-Optimization}

Referring to (1.2)-(1.4), it is clear that a class of reduced order controllers having the same dimension can be defined in terms of the sub-blocks of $j$ (s) and $Q(s)$, with $Q(s)$ constrained to be constant. These are parameterized in terms of a constant $Q$ matrix having the dimensions of the plant transfer function matrix. A search over all constant $Q$ can lead to improved reduced order controllers over that of the simplest case where $Q=0$ as in the previous subsection.

Such a search over constant $Q$ is relatively simple computationally compared to a search over the scale factors $D_{r}, D_{u}, D_{y}$ involving repeated application of the balanced realization algorithm.

The search over constant $Q(s)$ can be simplified exploiting the fact that all closed-loop transfer functions are affine in $Q(s)$ when $j(s)=J(s)$, so are "close" to affine in $Q(s)$ when $J(s)$ is "close" to $J(s)$.

2.4 Estimation-Based Reduction

Control schemes based on state estimate feedback can be viewed as an estimator/controller driven from both the plant inputs $u(t)$ and outputs $y(t)$ with an output $u(t)$. $\Lambda$ s depicted in Figure 2.2a, we can think of an augmented plant $G_{a}^{T}(s)=\left[G^{T}(s) I\right]$ with an augmented output $\left[y^{T}(t) u^{T}(t)\right]^{T}$ driving a controller, denoted $K_{a}(s)$. Now the corresponding $K_{a}(s)$ and $J_{a}(s)$ are given from

$$
J_{a}(s)=\left[\begin{array}{c|ccc}
A+H C & {[-(B+H D)} & H] & 0 \\
\hline-F & {[0} & 0] & I \\
C & {[-D} & I] & 0
\end{array}\right]_{T}
$$

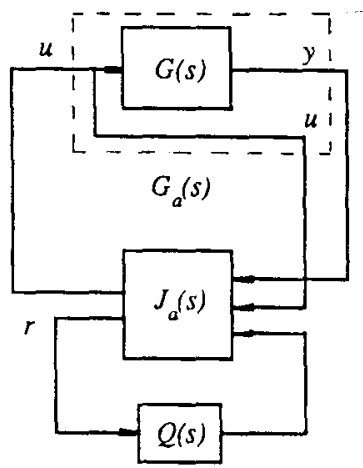

(a)

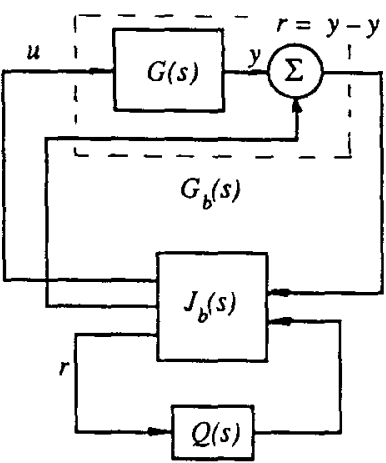

(b)
Figure 2.2 Estimation-based reduction

Notice that $J_{a}(s)$ is stable so that reduced or der approximations $j_{a}(s)$, - $\hat{K}_{a}(s)=\left[\hat{J}_{a}(s)\right]_{11}$ are also stable. There appears to be no other a priori guideline in selecting between reducing this controller and the conventional one. Clearly in any particular application one may be given a "better" reduced order performance and robustness. $A$ dual approach is to view the plant as in Figure 2.2b, where the plant $G_{b}(s)=[G(s) I]$ has an additional input which is added to the output of $G(s)$. The corresponding transfer function for $J_{b}(s)$ is given by

$$
J_{b}(s)=\left[\begin{array}{c|cc}
A+B F & H & -B \\
\hline-F & 0 & I \\
C+D F & 0 & -D \\
0 & I & 0
\end{array}\right]_{\tau}
$$

Comparison of $(2.13),(2.14)$ with (2.5) reveals that

$$
\begin{aligned}
& \bar{X}(s)=\left[\begin{array}{cc}
-I & 0 \\
0 & I
\end{array}\right] J_{a}(s)\left[\begin{array}{cc}
I & 0 \\
0 & I \\
-I & 0
\end{array}\right], \\
& \mathcal{X}(s)=\left[\begin{array}{ccc}
-I & 0 & 0 \\
0 & I & -I
\end{array}\right] J_{b}(s)\left[\begin{array}{cc}
0 & -I \\
-I & 0
\end{array}\right]
\end{aligned}
$$

This suggests the possibility of reducing $\bar{X}$ or $\mathcal{X}(s)$ as another method of controller reduction. The fractional decomposition of the reduced order controller would be found by applying standard model reduction methods to $\tilde{\mathcal{X}}$ or $\mathcal{X}(s)$.

2.5 Controller Reduction Maintaining Performance

Consider that a performance objective is to minimize the energy in some internal variable, or its estimate, denoted $e$. For the controller class (2.2), which can be interpreted as state estimate feedback, it is common for $e$ to be a linear combination of the states of $K^{*}(s)$. Thus here we assume that the transfer function from $u$ to $e$ is $E\left(s I-A^{*}\right)^{-1}(-H)$. Now, the augmentation of the $J(s)$ block of (2.4) to incorporate this transfer function is

$$
J_{e}(s)=\left[\begin{array}{c|cc}
A^{*} & -I J & B \\
\hline F & 0 & I \\
-(C+D F) & I & -D \\
E & 0 & 0
\end{array}\right]_{T}, K_{e}(s)=\left[J_{e}(s)\right]_{11}
$$

Scaling of this in terms of $D_{y}, D_{u}, D_{r}, D_{\text {, and }} D_{e}$ is now a natural extension of the scaling in (2.10). Likewise generalizations of $(2.11)$ to $j_{\text {scaled }}(s)$ and $\hat{K}(s)=D_{u}\left[\hat{J}_{\text {caled }}(s)\right]_{11} D_{y}^{-1}$ are straightforward. The relative significance of $D$, determines the emphasis on performance of the controller in the reduction process, and can be fine tuned by a trial and error procedure. 2.6 Frequency Shaped Reduction

Just as a frequency shaped reduction of $K(s)$ can lead to improved reduced order controllers, so a frequency shaped reduction of $J(s)$ leading to a reduced $\hat{h}(s)$ can give improvement. It might be that we require robustness in a frequency band only. That is, we require robustness to $Q(s) \in R H^{\infty}$ in this frequency band. Under such circumstances it makes sense to insert in Figure $1.1 \mathrm{~b}$ a stable band pass filter between the residuals $r(t)$ and the input to $Q(s)$, and require robustness to all $Q(s) \in R H^{\infty}$ as before. The band pass filter can be used as a frequency shaped augmentation of $J(s)$ being in series with $J_{21}(s)$ (or $J_{12}(s)$ ) and $J_{22}(s)$. A gain the augmented $J(s)$ can be reduced and the 11 -block extracted as a frequency shaped reduced controller $\hat{K}(s)$. The augmentation increases the degree of $J(s)$, while the following step reduces the degree of $J(s)$. In many cases, the effect of the errors introduced by increasing the degree of $J(s)$ in the intermediate step will be outweighed by the improved robustness of the closed loop controller.

Of course general frequency shapings can be employed based on the closed-loop transfer functions. In fact, it is sometimes impossible to obtain a good reduction of $J(s)$ unless frequency weighted reduction methods are used.

To avoid numerical difficulties when the combined order of $J(s)$ and any frequency shaping is high, it makes sense to first carry out a preliminary unweiglited reduction of $J(s)$ and any frequency shaping using balanced truncation. Such a reduction allows a degree reduction with relatively small error.

2.7 Generation of $F, H$

When the plant and controller have the same degree, but a selection $F$, $H$ to satisfy (2.2) is not known a priori, then such selections can be found for generic $K(s), G(s)[10]$. More precisely

Lemma 2.1 Consider the plant/controller pair $G(s), \bar{K}(s)$ with minimal $n$ th-order state-space realizations

$$
G(s)=\left[\begin{array}{l|l}
A & B \\
\hline C & D
\end{array}\right]_{T}, \bar{K}(s)=\left[\begin{array}{l|l}
\bar{A} & \ddot{B} \\
\hline \tilde{C} & 0
\end{array}\right]_{T}
$$

The controller can only be represented in the form (2.2) if and only if there exists a real, nonsingular solution $Z$ to the quadratic matrix equation

$$
A Z+B \bar{C}-Z \bar{B} C Z-Z(\bar{A}+\bar{B} D \bar{C})=0
$$

Moreover, when a real, nonsingular $Z$ exists

$$
F=\bar{C} Z^{-1}, \quad H=-Z \dot{B}
$$

Proof: The controller $\bar{h}(s)=\bar{C}(s I-\bar{A})^{-1} \tilde{B}$ has the same transfer function as $K(s)=-F\left(s I-A^{*}\right)^{-1} H$ of $(2.2)$ if and only if they are related by a similarity transformation

$$
Z \bar{A} Z^{-1}=A^{*}, Z \bar{B}=-H, \bar{C} Z^{-1}=F
$$

Algebraic manipulation with (2.20) leads directly to (2.18). In the SISO case solutions $F, H$ always exist [11] under

$[A, B],[\bar{A}, \bar{B}]$ controllable, $[A, C],[\bar{A}, \bar{C}]$ observable

Remarks:

(i) Sufficient conditions for multivariable $G(s), K(s)$ are presently under consideration.

(ii) There are in general a class of nonsingular solutions of (2.18), giving rise to a class of $J(s), j(s)$ and $K(s)$. For each $J(s)$ the bounds on $\|J(s)-j(s)\|$ will in general be different, and each approximation will have its own inherent frequency shaping. Clearly some selections of $J(s)$ will be better than others. This has been borne out with exampics 
studied, but as yet there is no elegant method to select the best $J(s)$ to use. 2.8 Staged Reduction

So far the simplest situation has been studied-namely when the degrees of $G(s), K(s)$ are the same. Should $K(s)$ be of a higher degree than $G(s)$ it makes sense to first perform a standard reduction of $K(s)$ until it is the same degree as $G(s)$. Such preliminary reduction can usually be made with negligible errors compared to subsequent reductions to achieve a lower degree estimates $\hat{K}(s)$. The same holds mutatis mutandis when $G(s)$ is of a ligher degree than $K(s)$.

2.9 Simultaneous Stabilization

Consider that $K$ is designed to give acceptable performance/robustness for a number of plants $G_{1}, G_{2} \ldots G_{N}$. Associated with each plant $G_{i}$ there is a corresponding $J_{i}$ with $\left[J_{i}\right]_{11}=K$ for each $i$. By bringing each $J_{i}$ to the same co-ordinate basis it is possible to define a block $\mathcal{J}(s), \mathcal{Q}(s)$ as in Figure 2.3 such that

$$
J_{i}=\left[\begin{array}{cc}
\mathcal{J}_{1,1} & \mathcal{J}_{1, i+1} \\
\mathcal{J}_{i+1,1} & \mathcal{J}_{i+1, i+1}
\end{array}\right]
$$

By setting $\mathcal{Q}_{k l}=0$ for $k, l \neq i, i=1,2 \ldots N$, the class of all stabilizing controllers for $G_{i}$ is characterized in terms of $Q_{i i} \in R H^{\infty}$. This leads to the following lemma.

LEMMA 2.2 With (2.22) holding, the class of all stabilizing controllers for $G_{i}$ for $i=1,2 \ldots N$ is a subset of the class of all controllers of Figure 2.2 with arbitrary $\mathcal{Q}(s) \in R H^{\infty}$

To achieve a reduced order controller $\hat{K}(s)$ for $K(s)$, we propose the reduction of $\mathcal{J}(s)$ giving $\hat{K}=[\hat{J}]_{11}$. When $N=1$, this method reduces to that presented previously. Scaling can be introduced to order the importance of the various plants $G_{i}$.

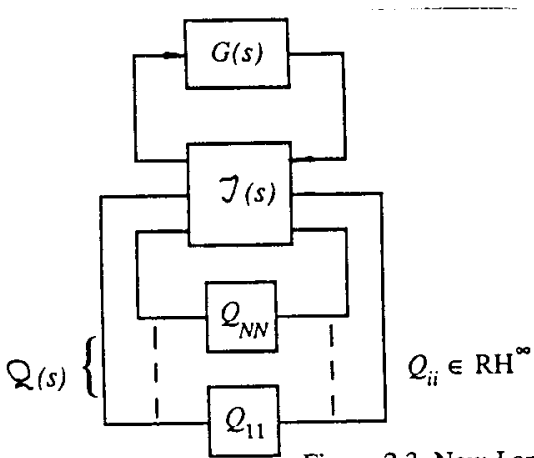

Figure 2.3 New J and Q blocks based on controller designed for simultaneous stabilization of many plants.

2.10 Reduced Order Plant

It may be that for simulation purposes a reduced order plant is required. In the reduction technique described above it is possible to extract a reduced order plant $\hat{G}(s)$ in addition to the reduced order controller as follows

$$
\hat{G}=\left[\begin{array}{c|c}
\widehat{A^{*}}-\hat{B} \hat{F}-\hat{H} \dot{C}-\hat{H} \hat{D} \hat{F} & \dot{B} \\
\hline \dot{C} & \hat{D}
\end{array}\right]_{T}
$$

where the estimates $\widehat{A^{*}}, \hat{H}, \hat{F}, \hat{C}$ and $\hat{D}$ are obtained from $\hat{J}$. One problem is that there is no guarantee that $\hat{K}$ close to $K$ will ensure that $\dot{G}$ will be close to $G$, or indeed, that $K$ or $\hat{K}$ will stabilize $\hat{G}$. Let us instead propose that a reduced order $\bar{G}$ be obtained from a dual procedure to that giving $\hat{K}$, so that at least $\hat{G}$ is close to $G$ and is stabilized by $K$. In the dual procedure the roles of $\mathrm{K}, \mathrm{G}$ are merely interchanged.

\section{RATIONALE}

\subsection{Preserving Robustness Properties}

The class of all stabilizing controllers for a plant $G(s) \in R_{p}$ sliall be denoted

$$
\mathcal{K}_{G} \triangleq\left\{K \in R_{p} \mid H(G, K) \in R H^{\infty}, \operatorname{det}(I-G K) \neq 0\right\}
$$

where $H(G, K)$ represents the closed loop transfer functions

$$
I I(G, K)=\left[\begin{array}{cc}
I+K(I-G K)^{-1} G & K(I-G K)^{-1} \\
(I-G K)^{-1} G & (I-G K)^{-1}
\end{array}\right]=\left[\begin{array}{cc}
I & -K \\
-G & I
\end{array}\right]^{-1}
$$

Such classes have a parametrization in terms of an arbitrary $Q \in R H^{\infty}$ and an abitrary factorization $K=U V^{-1} \in \mathcal{K}_{G}$

$\mathcal{K}_{G} \triangleq\left\{\mathcal{K}_{G}(Q)=(U+M Q)(V+N Q)^{-1} \mid Q \in R H^{\infty}, \operatorname{det}(V+M Q) \neq 0\right\}$

By duality, a controller $K(s) \in R_{p}$ stabitizes a class of plants $\mathcal{G}_{K}$, and the reduced order controller $\hat{K}$ stabilizes a class of plants $\mathcal{G}_{\dot{K}}$.

DEFINITION: The robustness properties of a stabilizing controller $K$ with respect to a plant class $\mathcal{G}^{\star}$ are said to be preserved in a controller reduction, yielding $\hat{K}$ when

$$
\mathcal{G}^{\star} \subset \mathcal{G}_{K, \dot{K}} \triangleq \mathcal{G}_{K} \cap \mathcal{G}_{\dot{K}}
$$

Remarks:

(i) $A$ dual definition of preserving robustness is as follows. With $\boldsymbol{\kappa}_{G}$ the class of all stabilizing controllers for a reduced order plant $\dot{G}$, the robustness properties of a plant $G$ with respect to a controller class $K^{*}$ are said to be preserved in a plant reduction yielding $\hat{G}$ when

$$
\mathcal{K}^{\star} \subset \mathcal{K}_{G, \dot{G}} \triangleq \mathcal{K}_{G} \cap \mathcal{K}_{\hat{G}}
$$

The class of stabilizing controllers for a plant $\hat{G}$ can similarly be param eterized in terms of $Q \in R H^{\infty}$.

$\mathcal{K}_{\dot{G}} \triangleq\left\{\mathcal{K}_{\hat{G}}(Q)=(\hat{U}+\hat{M} Q)(\hat{V}+\hat{N} Q)^{-1} \mid Q \in R H^{\infty}, \operatorname{det}(\hat{V}+\hat{N} Q)=0\right\}$

where $\hat{K}=\hat{U} \hat{V}^{-1}$ is a stabilizing reduced order controller for the reduced order plant $\dot{G}=\hat{N} \dot{M}^{-1}$.

(ii) A controller or plant reduction that preserves the robustness properties defined in (3.4), (3.5) should maintain $\mathcal{G}_{K, \dot{K}}$ close to $\mathcal{G}_{K}$ and $\mathcal{K}_{G, \dot{G}}$ close to $\mathcal{K}_{G}$. In other words, the reduction should give $\hat{K}$ such that

$$
\Delta \mathcal{G} \triangleq \mathcal{G}_{K} \Delta \mathcal{G}_{\bar{K}} \quad \text { or } \quad \Delta \mathcal{K} \triangleq \mathcal{K}_{G} \Delta \mathcal{K}_{\dot{G}} \text { is small } \dagger
$$

(iv) The fractional maps (3.3), (3.6) can be depicted as in Figure 3.1.
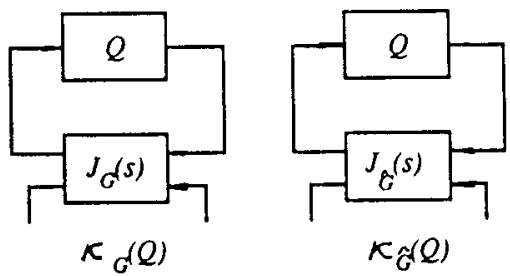

Figure 3.1 Linear fractional maps

\subsection{Closeness Measures}

Standard $L^{2}$ or $L^{\infty}$ norms define measures of closeness of $\mathcal{G}_{K}(Q)$ to $\mathcal{G}_{\hat{K}}(Q)$ for any specific $Q \in R H^{\infty}$, with such norms highly $Q$ dependent functions. The controller reduction method based on the reduction of $\bar{x}^{\prime}(s)$ or $J_{\mathrm{a}}(s)$ suggests convenient measures of closeness of the classes $\mathcal{G}_{K}, \mathcal{G}_{\dot{K}}$ being

$$
\|\Delta \bar{x}(s)\| \text { or }\left\|\Delta J_{a}(s)\right\| \quad \text { respectively }
$$

where $\Delta J_{a}(s)=J_{a}(s)-\hat{J}_{a}(s)$ etc.

The next lemma shows that a sufficient condition for the controller reduction objective that $\mathcal{G}_{K}(Q)$ is close to $\mathcal{G}_{\hat{K}}(Q)$ is that $\|\Delta \overrightarrow{\mathrm{x}}(s)\|$ or $\left\|\Delta J_{a}(s)\right\|$ in (3.8) be small. A dual argument can be developed for the corresponding plant reduction.

LEMma 3.1 With the definition (3.8) and

$$
\|\Delta \tilde{x}\| \leq \epsilon \text { or }\left\|\Delta J_{a}\right\| \leq \epsilon
$$

then for generic $Q \in R H^{\infty}$, as $\epsilon \rightarrow 0$

$$
\left\|\mathcal{G}_{K}(Q)-\mathcal{G}_{\dot{K}}(Q)\right\| \rightarrow 0 \text { with } \mathcal{O}(\epsilon)
$$

Proof: Observe that from (3.10)

$$
\begin{aligned}
\mathcal{G}_{\hat{K}^{\prime}}(Q)-\mathcal{G}_{K}(Q) & =(\hat{N}+\hat{V} Q)(\hat{M}+\hat{U} Q)^{-1}-(N+V Q)(M+U Q)^{-1} \\
& =\left\{\mathcal{G}_{K}(Q)[\Delta M+\Delta U Q]-[\Delta N+\Delta V Q]\right\}[\hat{M}+\hat{U} Q]^{-1}
\end{aligned}
$$

$\dagger$ Here the binary set operator $\Delta$ is the symmetric difference defined as $A \triangle B=(A \cap \bar{B}) \cup(\vec{A} \cap B)$. 
For generic $Q$ and with $\|\Delta, \bar{l}\| \leq \epsilon$, as $\Delta M, \Delta N, \Delta U, \Delta V \rightarrow 0$,

$\left\|\mathcal{G}_{\hat{K}}(Q)-\mathcal{G}_{K}(Q)\right\| \rightarrow 0$ with $\mathcal{O}(\Delta M, \Delta N, \Delta U, \Delta V)$ and the result $(3.10)$ follows. Since from (2.14) $\left\|\Delta J_{a}\right\| \leq \epsilon$ implies $\|\Delta \bar{x}\| \leq \sqrt{2} \epsilon$, then $\left\|\Delta J_{a}\right\| \leq \epsilon$ implies (3.10) also.

Remark:

By appropriate scaling, the controller reduction methods can be specialized to those of [13] involving only $\Delta \bar{U}, \Delta \bar{V}$. Clearly the methods proposed take into the account both the plant and the controller $\mathrm{dy}-$ nanics.

\section{EXAMPLES}

The method described in Sections 2.1-2.2 has been applied to the reduction of a 55th order LQG controller for an advanced active control research aeroplane [14], [15]. Figure 4.1 shows the block diagram of a flutter suppression and gust load alleviation design. The controls used are the elevator and the outboard aileron surfaces. Measurements of pitch rate and wing tip acceleration are used to estimate the aeroplane's rigid and elastic motion. Also shown in parentheses in Figure 4.1 are the root-mean-square responses, at various points in the control loop, to a $10 \mathrm{fts}^{-1}$ vertical Dryden turbulence. These values were used to scale $J_{\text {scaled }}(s)$ of $(2.10)$, and Hankel norm approximation was used to obtain the reduced order $J_{\text {acaled }}(s)$. Reduced order controllers of as low as fourth order could give a satisfactory closed loop performance.

Table 4.1 summarizes the results for different controllers, ranging from the original controller to the fourth order controller. Robustness properties have been evaluated based on single loop pliase and gain margins, and the worst-case stability margins have been recorded in the table. Note that the margins of stability have been preserved in accordance with the design requirements (gain margins of $6 \mathrm{~dB}$ and pliase margins of $30^{\circ}$ ). Similarly, the damping of the flutter mode always exceed the design requirement of 0.015 . Further reduction leads to an unstable closed-loop system. With other controller reduction methods such as modal residualization, the minimum order for the reduced order controller is ten. It is perceived that if the options described in Sections 2.3, 2.5-2.6 were considered, then further improvements in the controller reduction could be expected. This will be left for future work.

Table 4.1

Reduction of a 55 th order Flutter Suppression and Gust Load Alleviation Controller ${ }^{1}$

\begin{tabular}{clllll} 
Order & $\begin{array}{l}\text { Flutter } \\
\text { Node } \\
\text { Damping }\end{array}$ & $\begin{array}{l}\text { Stability } \\
\text { Margins }\end{array}$ & $\begin{array}{l}\text { Bending } \\
\text { Mornent } \\
\text { (in-lls) }\end{array}$ & $\begin{array}{l}\text { Shear } \\
\text { (lbs) }\end{array}$ & $\begin{array}{l}\text { Torsion } \\
\text { (in-lbs) }\end{array}$ \\
\hline 55 & 0.074 & $14.0 \mathrm{~dB}, 58.6^{\circ}$ & $2.348 \times 10^{5}$ & 854 & $\mathbf{4 . 4 3 7 \times 1 0 ^ { 4 }}$ \\
10 & 0.034 & $14.0 \mathrm{~dB}, 59.0^{\circ}$ & $2.593 \times 10^{5}$ & 890 & $4.200 \times 10^{4}$ \\
9 & 0.039 & $5.8 \mathrm{~dB}, 70.0^{\circ}$ & $2.318 \times 10^{5}$ & 859 & $4.495 \times 10^{4}$ \\
8 & 0.032 & $10.0 \mathrm{~dB}, 69.0^{\circ}$ & $2.010 \times 10^{5}$ & 930 & $4.821 \times 10^{4}$ \\
7 & 0.032 & $15.0 \mathrm{~dB}, 38.0^{\circ}$ & $2.345 \times 10^{5}$ & 862 & $4.779 \times 10^{4}$ \\
6 & 0.027 & $7.0 \mathrm{~dB}, 28.0^{\circ}$ & $2.362 \times 10^{5}$ & 871 & $4.968 \times 10^{4}$ \\
5 & 0.016 & $15.0 \mathrm{~dB}, 81.0^{\circ}$ & $2.371 \times 10^{5}$ & 997 & $7.117 \times 10^{4}$ \\
4 & 0.016 & $7.5 \mathrm{~dB}, 70.0^{\circ}$ & $2.080 \times 10^{5}$ & 1102 & $7.877 \times 10^{4}$
\end{tabular}

1 The bending monent, shear force, and torsion are root-inean-square re sponses to a $10 \mathrm{fts}^{-1}$ vertical Dryden turbulence.
We will now make some remarks on a second example, one which is well studied in the literature [5], [6], [13]. Our aim here is not to demonstrate the superiority of our various methods, since the inbuilt frequency weighting in the reduction technique of [6] turns out to be highly suited to this example; a simple application of our methods does not do as well. Rather, our aim is to be convinced that the methods here can be competitive, depending on the engineering criteria for judging robustness/performance. Indeed, for a frequency weighted version of our technique we claim equality with, and perhaps marginal superiority to, some of the nuethods of [13]

An eighth-order controller is reduced to a fifth-order controller using various controller reduction methods. The original plant and controller are given in $[5]$ (case $q=100$ ). The plant has one rigid body mode and three lightly damped structural modes $(\zeta=0.02)$. The conmand response corresponding to the full order controller does not exhibit any lightly damped structural modes. This is due to the fact that with precisely placed notch filters in the feedback controller, the residues at the structural mode frequencies are negligible. Reduction of the controller order alters the location of the notch filter poles and zeros, and may introduce large residues at the uncontrolled structural modes.

Reduction of a stable right coprine factorization of the controller [6] produces a good approximation in the low frequency region, and introduces residual responses at the second structural mode frequency. Comparison of the frequency weighted balanced truncation method of [5] with other reduction methods is given in [6], [13]. Frequency weighted balanced truncation is here applied to the controller structure $J_{e}(s)$ described in previously (see Figure 4.2). The criterion function in the cost function is appended to the outputs of $J_{e}(s)$ to maintain performance. The scale factors applied at the inputs and outputs of $J_{e}(s)$ are determined by evaluating the closed-loop covariance responses. This reduction technique yields a reduced order controller with good robust ness/performance properties in a systematic fashion, with fewer iterations than the method of [13]. (In [13], an augmented output is also included in the reduction to improve performance.)

For comparative purposes a reduced order controller, obtained by direct optimization via the SANDY design algorithm [16], has also been studied. The reduction based on the same cost objective, process noise, and sensor noise characteristics. The resulting step response agrees closely with the original design. The low order controller has the advantage that it only excites the plant at the first structural mode frequency.

\section{CONCLUSIONS}

A class of controller reduction methods have been proposed which preserve the robustness and performance qualities of the controller. The methods can be viewed as consisting of three steps. The first is organizing the plant and controller information. The second is applying standard model reduction techniques, and the third is extracting and re-optimizing (if necessary) a reduced order controller from the second step results using a constant stabilizing controller structure. Trade-offs between performance and robustness can be achieved by scaling, and indecd by certain extreme scalings, other metiods in the literature can be recovered.

The proposed methods are, in the first instance, most appropriate for controller designs organized as state estimate feedback sclıemes. However all stabilizing controller designs can be organized as such [12], and our methods do extend of stabilizing controllers. Simulation studies have supported the rationale for the methods proposed.

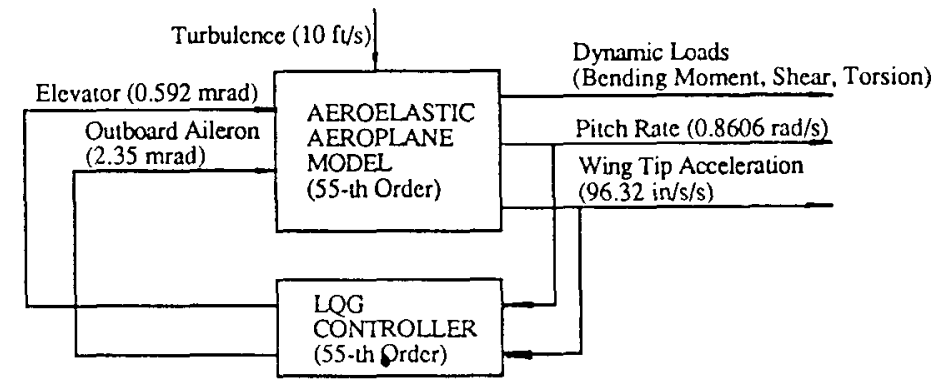

Figure 4.1 Block diagram of the flutter suppression and gust load alleviation system. 


\section{REFERENCES}

[1] K. Glover, "All Optimal IIankel Norm Approximations of Linear Mlultivariable Systems and their $L^{\infty}$ - Error Bounds", Int. J. Control, Vol. 39, No.6, pp.1115-1193, 1984.

[2] B.C. Moore, "Principal Component Analysis in Linear Systems: Controllability, Observability, and Model Reduction," IEEE Trans. Automat. Control, Vol. AC-26, No.1, pp.17-32, February 1981.

[3] G.A. Latham and B.D.O. Anderson, "Frequency-Weighted Optimal Hankel Norm Approximation of Stable Transfer Functions," Syst. Control Lett., Vol.5, pp.229-236, February 1985.

[4] B.D.O. Anderson, "Weighted Hankel Approximation: Calculation of Bounds," Syst. Control Lett., Vol.7, pp.247-255, July 1986.

[5] D. Enns, Model Reduction for Control System Design, Ph.D. Thesis, Dept. of Aeronautics and Astronautics, Stanford University, June 1984

[6] Y. Liu and B.D.O. Anderson, "Controller Reduction via Stable Factorization and Balancing," Int. J. Control, Vol.44, No.2, pp.507-531,1986.

[i] J.A. Davis and R.E. Skelton, "Another Balanced Controller Reduction Algorithm," Syst. Control Lett., Vol.4, pp.79-83, A pril 1984.

[8] C.N. Nett, C.A. Jacobson, and M.J. Balas, "A Connection between State-Space and Doubly Coprime Fractional Representations," IEEE Trans. Automat. Control, Vol. AC-29, pp.831-832, September 1984.

[9] D.C. Youla, H. Jabr and J.J. Biongiorno, "Modern Wiener-Hopf Design of Optimal Controllers,Part H: The Multivariable Case," IEEE Trans. Automat. Contral, Vol.21, pp.319-338, June 1076.

[10] I1.-B. Meyer, "The Matrix Equation $A Z+B-Z C Z-Z D=0$," SIAMI J. Appl. Math., Vol. 20, No. 1, January1976.

[11] D.J. Clements and B.D.O. Anderson, "Polynomial Factorization via the Riccati Equation", SIAMI J. Appl. Math., Vol. 31, No. 1, July 1976.

[12] J.B. Moore, K. Glover, and A.J. Telford, "On the Class of All Stabilizing Controllers," Proceedings of the 25th IEEE Conference on Decision and Control, December 10-12, 1986, pp.693-698.

[13] Y. Liu and B.D.O. Anderson, "Controller Reduction: Concepts and APproaches," Proceedings of the American Control Conference, Minnesota, U.S.A., 1987, pp.1-9.

[14] "Integrated Application of Active Controls (IAAC) Technology to an Advanced Subsonic Transport Project: Current and Advanced ACT Control System Definition Study - Volume I," NASA Contractor Report 165631, Final Report, October 1981.

[15] Yi Liu, Brian D.O. Anderson, and Uy-Loi Ly, "Coprime factorization controller reduction with Bezout identity induced frequency weighting," submitted for publication.

[16] Uy-Loi Ly, "A Design Algorithm for Robust Low-Order Controllers," Stanford University, Department of Aeronautics and Astronautics, Report No. 536, November 1982.

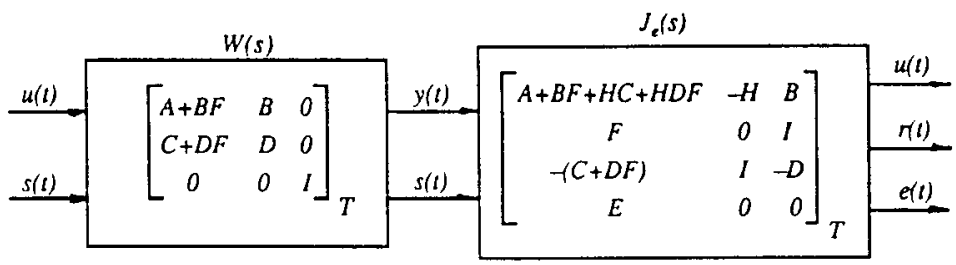

Figure 4.2 Frequency-weighting at the controller inputs. 